\title{
Should Structure Functions Be Used to Estimate Power Laws in Turbulence? A Comparative Study
}

\author{
Gerald A. Nichols-Pagel \\ Department of Mechanical Engineering, Box 356200, University of Washington, \\ Seattle, Washington 98195-6200
}

Donald B. Percival

Applied Physics Laboratory, Box 355640, University of Washington, Seattle, Washington 98195-5640

Per G. Reinhall and James J. Riley

Department of Mechanical Engineering, Box 356200, University of Washington, Seattle, Washington 98195-6200

\begin{abstract}
Second order structure functions are widely used to characterize turbulence in the inertial range because they are simple to estimate, particularly in comparison to spectral density functions and wavelet variances. Structure function estimators, however, are highly autocorrelated and, as a result, no suitable theory has been established to provide confidence intervals for turbulence parameters when determined via regression fits in $\log / \log$ space. Monte Carlo simulations were performed to compare the performance of structure function estimators of turbulence parameters with corresponding multitaper spectral and wavelet variance estimators. The simulations indicate that these latter estimators have smaller variances than estimators based upon the structure function. In contrast to structure function estimators, the statistical properties of the multitaper spectral and wavelet variance estimators allow for the construction of confidence intervals for turbulence parameters. The Monte Carlo simulations also confirm the validity of the statistical theory behind the multitaper spectral and wavelet variance estimators. The strengths and weaknesses of the various estimators are further illustrated by analyzing an atmospheric temperature time series.
\end{abstract}




\section{Introduction}

In his classic work Kolmogorov [11] theorized that, at turbulent scales too small to be directly affected by the energetic motions and too large to be affected by viscosity, the longitudinal and transverse velocity structure functions should only depend on the kinetic energy dissipation rate. This theory results in a statistical description of turbulence in which the structure function and related second order descriptors such as the spectral density function (SDF) and wavelet variance are linear on $\log / \log$ plots. In particular, the SDF is proportional to $|f|^{\alpha}$, where the constant of proportionality is related to the kinetic energy dissipation rate, and the power law exponent is $\alpha=-5 / 3$. Corrsin [5] and Obukhov [14] showed independently that the fluctuations of a passive scalar in a turbulent flow should exhibit this same power law behavior with a constant of proportionality that is related to the scalar dissipation rate in addition to the kinetic energy dissipation rate.

Experiments in turbulence often rely on estimates of the power law exponent $\alpha$ to identify the inertial subrange, in order to check for consistency with Kolmogorov-Obukhov-Corrsin (KOC) turbulence, and to test hypotheses concerning corrections to turbulence theory. Estimates of the constant of proportionality are important in estimating kinetic energy and scalar dissipation rates from environmental and laboratory flows. Estimation of the exponent and the constant of proportionality is commonly based upon the log of either a structure function estimator, an SDF estimator or a wavelet variance estimator. It is thus important to understand the statistical merits of the estimators used to characterize measured turbulence. As we demonstrate via examples in Section 2, a typical estimate of the structure function appears to be markedly more stable than certain corresponding SDF estimates. We demonstrate in this paper (Section 3) that the apparently superior stability of structure function estimates does not necessarily translate into estimates of the exponent and constant of proportionality that are superior to those from suitably chosen SDF and wavelet variance estimates. Moreover, while there is an appealing statistical theory for both SDF and wavelet variance estimators that makes it possible to ascertain the amount of uncertainty in the resulting estimates of the exponent and constant of proportionality, the same does not hold for structure function estimators, for which the sampling theory is quite complicated [7]. See Muzy et al. [13] for more on fundamental drawbacks of the structure function in comparison to wavelet transforms.

The remainder of this paper is organized as follows. After a motivating illustration of the relative merits of structure function and SDF estimators, we define - and consider the basic properties of - estimators for the exponent and the constant of proportionality based upon a multitaper SDF estimator, a structure function estimator and wavelet variance estimators. In Section 3 
we use computer experiments to verify the statistical theory for the multitaper and wavelet-based estimators and to compare these estimators to the structure function-based estimators. In Section 4 the estimators are applied to an atmospheric temperature signal. Section 5 summarizes our results and recommendations.

\section{Background}

As a motivating example, Figure 1 compares two approaches for estimating the exponent $\alpha$ of a process with an SDF that is proportional to a power law $|f|^{\alpha}$ over two decades of frequencies. The left-hand column shows two realizations of a stationary process $\left\{X_{t}\right\}$, with an SDF given by $5 f^{-5 / 3}$ for $0.0025 \leq f \leq 0.25$. The middle column shows two multitaper SDF estimates (Section 2.1), along with least squares lines fitted in $\log / \log$ space over the frequency range exhibiting the $|f|^{-5 / 3}$ behavior (the lines are displaced upwards on the plot to make them easier to see). The right hand column shows corresponding plots for structure function estimates (Section 2.2), again with lines two decades in length that represent the theoretical slope and extent of the power law behavior in the simulated series. The multitaper spectral estimates $\hat{S}_{X}^{(m t)}$ appear very noisy compared to the structure function estimates $\hat{D}_{X}$. In addition, the proportion of explained variation, $R^{2}$, is substantially higher for the structure function estimates. One might conclude that the structure function is the superior estimate here; however, the multitaper estimates of the power law exponent $\alpha \doteq-1.667$ and constant of proportionality $B_{1}=5$ (derived from the slopes and intercepts of the fitted lines) are closer to the true values. In fact, the smoothness of the structure function is the result of a high degree of correlation between structure function estimates that are separated by small to moderate distances. This smoothness masks the variability in the estimated power law exponent and constant of proportionality and leads to the large $R^{2}$ statistic for the linear regression. The multitaper estimates, in contrast, rapidly decorrelate with increasing frequency separation; the variability in the estimates is clearly observed. As we demonstrate in what follows, the covariance structure of the multitaper estimate is much simpler, and can be approximated well independently of the data to quantify the variability of the estimated exponent and constant of proportionality and to establish confidence intervals for these parameters.

\subsection{Multitaper Spectral Estimation}

Multitaper spectral estimation is a technique introduced by Thomson [19] that yields a largely unsmoothed or 'raw' estimator with both low bias and 

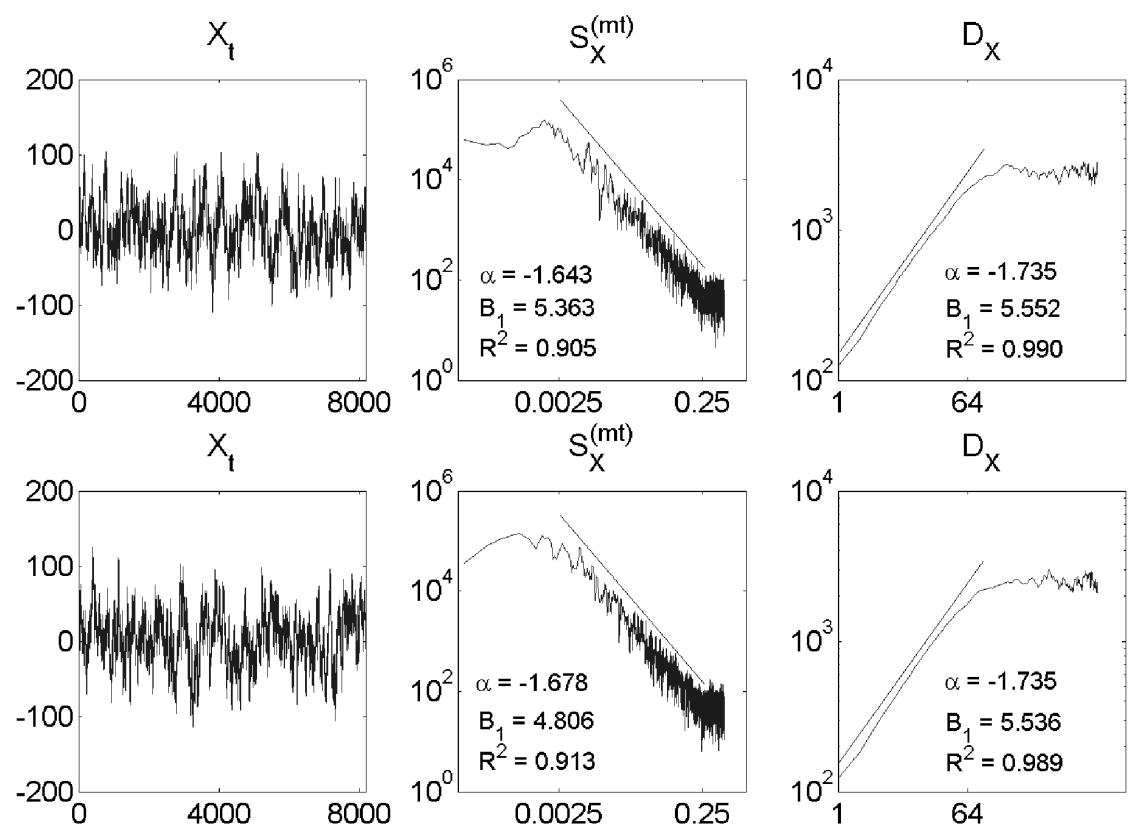

Fig. 1. Comparison of multitaper spectral and structure function estimates

tractable covariance and distributional properties. Multitapering builds upon the notion of a single data taper, which has been used routinely since the 1950s to reduce the bias in spectral estimators. In what follows, we first review how a single data taper is used to form a spectral estimator, after which we define the multitaper spectral estimator $\hat{S}_{X}^{(m t)}$. We then proceed as in McCoy et al. [12] to formulate an estimator of the exponent of power law processes that is based upon $\hat{S}_{X}^{(m t)}$ and that is attractive when compared to competing methods.

Suppose we have a time series that can be regarded as a realization of one portion $X_{0}, \ldots, X_{N-1}$ of a stationary process with $\mathrm{SDF} S_{X}$ (for simplicity, we assume that $\left\langle X_{t}\right\rangle=0$, i.e., that the expected value of the process is zero; if this is not a reasonable assumption, the common practice is to replace $X_{t}$ by $X_{t}-\bar{X}$ in what follows, where $\bar{X}$ is the sample mean of the time series). A direct spectral estimator of $S_{X}$ is obtained by computing the magnitude squared of the Fourier transform of the product of the series $\left\{X_{t}\right\}$ and a suitable data taper $\left\{h_{t}\right\}$ :

$$
\hat{S}_{X}^{(d)}(f)=\left|\sum_{t=0}^{N-1} h_{t} X_{t} e^{-i 2 \pi f t}\right|^{2}, \quad|f| \leq \frac{1}{2} .
$$

The purpose of using the data taper is to obtain an estimator that is approximately unbiased; i.e., $\left\langle\hat{S}_{X}^{(d)}(f)\right\rangle \approx S_{X}(f)$. Tapering typically 'shrinks' the values of $X_{t}$ near the beginning and end of the time series toward zero, which effectively causes a loss of information that manifests itself as an increase in the variance of subsequently smoothed estimators. 
Multitapering is designed to recover some of the information lost by a single data taper while simultaneously maintaining low bias and decreasing variability to some degree. A multitaper estimator is created by averaging $K$ direct spectral estimators:

$$
\hat{S}_{X}^{(m t)}(f)=\frac{1}{K} \sum_{k=0}^{K-1} \hat{S}_{X, k}^{(d)}(f),
$$

where $\hat{S}_{X, k}^{(d)}$ is the direct spectral estimator obtained using the $k$ th data taper $\left\{h_{k, t}\right\}$. The data tapers are chosen so that each generates a direct spectral estimator with good bias properties, and each is approximately uncorrelated with the other $K-1$ spectral estimators. The average of these direct spectral estimators will have good bias properties and a smaller variance than any individual estimator $\hat{S}_{X, k}^{(d)}$. The individual estimators will be nearly uncorrelated if the data tapers are orthogonal [16]:

$$
\sum_{t=0}^{N-1} h_{j, t} h_{k, t}=0 \text { for } j \neq k .
$$

Riedel and Sidorenko [18] suggest using sine tapers:

$$
h_{k, t}=\left(\frac{2}{N+1}\right)^{1 / 2} \sin \left(\frac{(k+1) \pi(t+1)}{N+1}\right) \text {. }
$$

In the inertial range, the SDF of velocity for KOC turbulence has a power law behavior:

$$
S_{X}(f)=C \epsilon^{2 / 3}|f|^{-5 / 3},
$$

where $C$ is a universal constant and $\epsilon$ is the kinetic energy dissipation rate. On a logarithmic scale, the SDF is linear in frequency with an intercept that is related to the kinetic energy dissipation rate:

$$
\log \left(S_{X}(f)\right)=\log \left(C \epsilon^{2 / 3}\right)-\frac{5}{3} \log (|f|) .
$$

More generally, for an SDF of the form

$$
S_{X}(f)=B_{1}|f|^{\alpha}
$$

we can write

$$
\log \left(S_{X}(f)\right)=\log \left(B_{1}\right)+\alpha \log (|f|) .
$$

According to Walden et al. [21], the log multitaper estimator can be written as the sum of two components. The first is nonstochastic and is the true log spectrum plus a known constant, $\psi(K)-\log (K)$, where $\psi$ is the digamma function. The second is stochastic noise $\eta_{j}$ whose distribution is dictated by that of a $\log \left(\chi_{2 K}^{2}\right)$ random variable. Hence $\eta_{j}$ has a known variance $\sigma_{\eta}^{2}=$ $\psi^{\prime}(K)$, where $\psi^{\prime}$ is the trigamma function (if $K$ is about 5 or greater, the 
distribution of $\eta_{j}$ is approximately Gaussian). Following McCoy et al. [12], we can estimate $\alpha$ and $B_{1}$ by using the linear regression

$$
Y_{j}=c+\alpha x_{j}+\eta_{j}, \quad j=1, \ldots, M,
$$

where $Y_{j} \equiv \log \left\{\hat{S}^{(m t)}\left(f_{j}\right)\right\}, c \equiv \log \left(B_{1}\right)+\psi(K)-\log (K), x_{j} \equiv \log \left(f_{j}\right)$ and $M$ is the number of frequency ordinates used in the regression over a range of positive Fourier frequencies $f_{j}$. In matrix form we have

$$
\mathbf{Y}=\mathbf{X} \Theta+\mathbf{N}
$$

where $\mathbf{Y}$ is the column vector of $Y_{j}$ variables, $\mathbf{X}$ is a matrix with ones in the first column and the $x_{j}$ variables in the second column, $\Theta=[c, \alpha]^{T}$, and $\mathbf{N}$ is the column vector of the $\eta_{j}$ variables. The corresponding least squares estimator is

$$
\hat{\boldsymbol{\Theta}}=\left[\mathbf{X}^{T} \mathbf{X}\right]^{-1} \mathbf{X}^{T} \mathbf{Y}
$$

with covariance matrix

$$
\left\langle[\hat{\boldsymbol{\Theta}}-\boldsymbol{\Theta}][\hat{\boldsymbol{\Theta}}-\boldsymbol{\Theta}]^{T}\right\rangle=\left[\mathbf{X}^{T} \mathbf{X}\right]^{-1} \mathbf{X}^{T} \boldsymbol{\Sigma} \mathbf{X}\left[\mathbf{X}^{T} \mathbf{X}\right]^{-1}
$$

Here $\boldsymbol{\Sigma}$ is the symmetric Toeplitz covariance matrix for $\mathbf{N}$. Following Walden et al. [21], the $(j, j+\tau)$ th element of the covariance matrix for $\mathbf{N}$ can be modeled by

$$
s_{\eta, \tau} \equiv \operatorname{cov}\left\{\eta_{j}, \eta_{j+\tau}\right\} \approx \begin{cases}\sigma_{\eta}^{2}\left(1-\frac{|\tau|}{K+1}\right), & \text { if }|\tau| \leq(K+1) \\ 0, & \text { otherwise }\end{cases}
$$

\subsection{Structure Function Estimation}

Kolmogorov's original hypothesis [11] was formulated in terms of the second order structure function:

$$
D_{X}(\tau)=\left\langle\left(X_{t+\tau}-X_{t}\right)^{2}\right\rangle
$$

For a process with an SDF given by Equation 1 with $-3 \leq \alpha<-1$, the structure function takes the form [22]

$$
D_{X}(\tau)=B_{2}|\tau|^{-(\alpha+1)}, \text { where } B_{2}=\frac{B_{1}}{(2 \pi)^{\alpha} \Gamma(-\alpha) \sin (-\pi(\alpha+1) / 2)} \text {. }
$$

After taking the logarithm of both sides, we obtain for positive $\tau$

$$
\log \left(D_{X}(\tau)\right)=\log \left(B_{2}\right)-(\alpha+1) \log (\tau)
$$


This relationship can be used to estimate $\alpha$ and $B_{2}$ by performing a regression involving the usual structure function estimator, namely,

$$
\hat{D}_{X}(\tau)=\frac{1}{N-\tau} \sum_{t=0}^{N-1-\tau}\left(X_{t+\tau}-X_{t}\right)^{2}
$$

where $N-\tau$ is the number of pairs of sample points separated by the positive distance $\tau$. The regression model in this case is

$$
Y_{\tau}=\log \left(B_{2}\right)-(\alpha+1) x_{\tau}+\eta_{\tau}
$$

where $Y_{\tau} \equiv \log \left(\hat{D}_{X}(\tau)\right), x_{\tau} \equiv \log (\tau)$, and the expected value of the error term $\eta_{\tau}$ is assumed to be zero. In contrast to 3 , the covariance matrix is difficult to determine because the error terms are highly correlated, with the correlation structure being model dependent. Genton [8] approximates this covariance matrix using an explicit formula in the situation where the data are independent. It is not clear that this approach is suitable for turbulence or other highly correlated processes.

\subsection{Wavelet Variance Estimation}

The utility of wavelet transforms for the characterization of power law processes has been well documented; see, e.g., [1] and [2]. Vidakovic [20] provides good background information on wavelet transforms.

Given a process $\left\{X_{t}\right\}$ that is either stationary or whose increments of a certain order form a stationary process, we can use a $j$ th level wavelet filter $\left\{\tilde{h}_{j, l}, j=\right.$ $\left.0,1, \ldots, L_{j}-1\right\}$ to define a wavelet coefficient process

$$
\bar{W}_{j, t} \equiv \sum_{l=0}^{L_{j}-1} \tilde{h}_{j, l} X_{t-l} \text { for } j=1,2,3, \ldots
$$

This process is associated with changes in co-located averages in $\left\{X_{t}\right\}$ on a standardized scale of $\tau_{j}=2^{j-1}$. Under mild conditions (see Percival and Walden [17] for details), the process $\left\{\bar{W}_{j, t}\right\}$ is stationary with mean zero. The wavelet variance is defined to be the variance of $\bar{W}_{j, t}$ :

$$
\nu_{X}^{2}\left(\tau_{j}\right)=\left\langle\bar{W}_{j, t}^{2}\right\rangle
$$

This variance offers a decomposition of the variability in $\left\{X_{t}\right\}$ over temporal or spatial scales. If $\left\{X_{t}\right\}$ is stationary with variance $\sigma_{X}^{2}$, then

$$
\sum_{j=1}^{\infty} \nu_{X}^{2}\left(\tau_{j}\right)=\sigma_{X}^{2}
$$


on the other hand, if $\left\{X_{t}\right\}$ is nonstationary but has stationary increments of a certain order, then

$$
\sum_{j=1}^{\infty} \nu_{X}^{2}\left(\tau_{j}\right)=\infty
$$

Given $X_{0}, X_{1}, \ldots, X_{N-1}$, the $j$ th level maximal overlap discrete wavelet transform (MODWT) wavelet coefficients are found by circular convolution with $\left\{\tilde{h}_{j, l}\right\}$ :

$$
\widetilde{W}_{j, t} \equiv \sum_{l=0}^{L_{j}-1} \tilde{h}_{j, l} X_{t-l \bmod N}, \quad t=0,1, \ldots, N-1 .
$$

The wavelet variance is estimated by computing the sample variance of the MODWT wavelet coefficients:

$$
\tilde{\nu}_{X}^{2}\left(\tau_{j}\right) \equiv \frac{1}{N} \sum_{t=0}^{N-1} \widetilde{W}_{j, t}^{2}
$$

The estimate $\tilde{\nu}_{X}^{2}\left(\tau_{j}\right)$ is referred to as the biased estimate of the wavelet variance because, whereas $\widetilde{W}_{j, t}=\bar{W}_{j, t}$ and hence $\left\langle\widetilde{W}_{j, t}^{2}\right\rangle=\nu_{X}^{2}\left(\tau_{j}\right)$ when $L_{j}-1 \leq t \leq$ $N-1$, the same does not necessarily hold when $0 \leq t<L_{j}-1$. Excluding these latter coefficients gives an unbiased estimator of the wavelet variance:

$$
\hat{\nu}_{X}^{2}\left(\tau_{j}\right) \equiv \frac{1}{M_{j}} \sum_{t=L_{j}-1}^{N-1} \widetilde{W}_{j, t}^{2}=\frac{1}{M_{j}} \sum_{t=L_{j}-1}^{N-1} \bar{W}_{j, t}^{2},
$$

where $M_{j} \equiv N-L_{j}+1$.

Percival and Walden [17] show that the wavelet variance can be related to the SDF because the $j$ th level MODWT wavelet filter acts like a band-pass filter with pass-band

implying that

$$
\frac{1}{2^{j+1}}<|f| \leq \frac{1}{2^{j}}
$$

$$
\nu_{X}^{2}\left(\tau_{j}\right) \approx 2 \int_{1 / 2^{j+1}}^{1 / 2^{j}} S_{X}(f) d f
$$

where the factor of two comes into play because we use SDFs that are assumed to be two-sided. Substitution of Equation 1 gives:

$$
\nu_{X}^{2}\left(\tau_{j}\right) \approx 2 B_{1} \int_{1 / 2^{j+1}}^{1 / 2^{j}} f^{\alpha} d f=B_{3} \tau_{j}^{-(\alpha+1)}
$$

where

$$
B_{3} \equiv \frac{2 B_{1}\left(2^{-(\alpha+1)}-4^{-(\alpha+1)}\right)}{\alpha+1} .
$$

After taking the logarithm of both sides, we obtain

$$
\log \left(\nu_{X}^{2}\left(\tau_{j}\right)\right) \approx \log \left(B_{3}\right)-(\alpha+1) \log \left(\tau_{j}\right)
$$


which is quite similar to Equation 4 involving the structure function.

Percival and Walden [17] formulate an estimator for $\alpha$ and $B_{3}$ by regressing the logarithm of the wavelet variance estimates against the logarithm of the wavelet scale:

$$
Y\left(\tau_{j}\right)=\zeta+\beta \log \left(\tau_{j}\right)+e_{j}
$$

where

$$
\begin{gathered}
Y\left(\tau_{j}\right) \equiv \log \left(\nu_{X}^{2}\left(\tau_{j}\right)\right)-\psi\left(\frac{\eta_{j}}{2}\right)+\log \left(\frac{\eta_{j}}{2}\right), \\
\zeta \equiv \log \left(B_{3}\right)=\log \left(\frac{2 B_{1}\left(2^{-(\alpha+1)}-4^{-(\alpha+1)}\right)}{\alpha+1}\right),
\end{gathered}
$$

$\beta \equiv-(\alpha+1), e_{j}$ has zero mean and variance $\psi^{\prime}\left(\frac{\eta_{j}}{2}\right)$, and $\eta_{j}$ is the 'equivalent degrees of freedom' for a scaled chi-squared distribution [17]. We estimate $\eta_{j}$ using the following relationship from Percival and Walden [17]:

$$
\eta_{j}=\max \left\{M_{j} / 2^{j}, 1\right\} .
$$

We can obtain estimates of $\beta$ and $\zeta$ using ordinary least squares; more appropriately, since the variance of the error term grows with increasing $j$, we can use weighted least squares with weights $w_{j}=1 / \psi^{\prime}\left(\frac{\eta_{j}}{2}\right)$. The ordinary least squares estimator takes the same form as the multitaper estimator with $Y_{j}=Y\left(\tau_{j}\right)$ and $x_{j}=\log \left(\tau_{j}\right)$. The corresponding weighted least squares estimator is

$$
\hat{\boldsymbol{\Theta}}=\left[\mathbf{X}^{T} \boldsymbol{\Sigma}^{-1} \mathbf{X}\right]^{-1} \mathbf{X}^{T} \boldsymbol{\Sigma}^{-1} \mathbf{Y},
$$

with covariance matrix

$$
\left\langle[\hat{\boldsymbol{\Theta}}-\boldsymbol{\Theta}][\hat{\boldsymbol{\Theta}}-\boldsymbol{\Theta}]^{T}\right\rangle=\left[\mathbf{X}^{T} \boldsymbol{\Sigma}^{-1} \mathbf{X}\right]^{-1},
$$

where $\boldsymbol{\Theta}=[\zeta, \beta]^{T}$ and $\boldsymbol{\Sigma}$ is a diagonal matrix with elements $\psi^{\prime}\left(\frac{\eta_{j}}{2}\right)$. Jensen [10] shows that the approximation for the slope in Equation 7 becomes exact as $j \rightarrow \infty$ and is quite reasonable even when $j \geq 2$ or 3 . The fitness of the approximation for the intercept will be evaluated, by numerical integration of the product of the true SDF and the wavelet squared gain function, in Section 3.

\subsection{Scaling range averaging for the estimation of $B_{1}$}

In turbulent signals, the constant of proportionality in the power law model is often used as a surrogate for the energy of the signal contained in the inertial range. It is through this interpretation that a relationship between the constant of proportionality and the dissipation rate is formed. In the previous sections we have outlined methods for estimating $B_{1}$ either directly or indirectly via $B_{2}$ 
or $B_{3}$ using, respectively, the structure function or wavelet variance; however, if the slope of the line for this intercept is not known, the intercept, by itself, does not indicate the signal energy contained within any given band of frequencies or any range of scales.

Here we consider an alternative estimator for $B_{1}$ that can be more readily interpreted in terms of dissipation rate. Note that the expected values of the $\log / \log$ models 2,5 , and 8 for the multitaper, structure function, and wavelet variance cases all take the form $b+\gamma x$ over an appropriate scaling region $x_{1} \leq x \leq x_{2}$. The average value of the $\log / \log$ model over this region is given by

$$
A \equiv \frac{1}{x_{2}-x_{1}} \int_{x_{1}}^{x_{2}} b+\gamma d x=b+\frac{\gamma}{2}\left(x_{1}+x_{2}\right) .
$$

In the multitaper case, $b=\log \left(B_{1}\right)+\psi(K)-\log (K)$, and $\gamma=\alpha$, so we can estimate $B_{1}$ (assuming $\alpha=-5 / 3$ ) using

$$
\hat{\hat{B}}_{1}^{(m t)}=\exp \left\{\hat{A}^{(m t)}+\frac{5}{6}\left(x_{1}+x_{2}\right)-\psi(K)+\log (K)\right\},
$$

where $\hat{A}^{(m t)}$ is formed by taking the average values of $Y_{j}=\log \left\{\hat{S}^{(m t)}\left(f_{j}\right)\right\}$ over the region of frequencies for which the power law model is assumed to hold. Based upon similar averages $\hat{A}^{(s f)}$ and $\hat{A}^{(w v)}$ for the structure function and wavelet variance cases, we obtain

$$
\hat{\hat{B}}_{1}^{(s f)}=(2 \pi)^{-5 / 3} \Gamma(5 / 3) \sin (\pi / 3) \exp \left\{\hat{A}^{(s f)}-\frac{1}{3}\left(x_{1}+x_{2}\right)\right\}
$$

and

$$
\hat{\hat{B}}_{1}^{(w v)}=-\frac{1}{3\left(2^{2 / 3}-4^{2 / 3}\right)} \exp \left\{\hat{A}^{(w v)}-\frac{1}{3}\left(x_{1}+x_{2}\right)\right\} .
$$

The estimators of $B_{1}$ obtained through this procedure will reflect the energy content of the chosen scaling range, regardless of the slope, but will only reflect the intercept of the regression line if the estimated value for $\alpha$ is $-5 / 3$. This is preferable to the alternative, namely, that the estimate of $B_{1}$ reflects the intercept of the regression line, regardless of the slope, and only reflects the energy content of the scaling range when the $\alpha$ estimate is $-5 / 3$.

\section{Results}

To compare the relative merits of the estimators described in the previous section, we consider a Gaussian stationary process with an SDF defined over 
$-0.5 \leq f \leq 0.5$ such that

$$
S(f)= \begin{cases}5 \times 0.0025^{-5 / 3}, & \text { for } 0 \leq|f| \leq 0.0025 \\ 5|f|^{-5 / 3}, & \text { for } 0.0025<|f| \leq 0.25 ; \text { and } \\ 5 \times 0.25^{-5 / 3}, & \text { for } 0.25<|f| \leq 0.5\end{cases}
$$

This SDF has power law behavior $B_{1}|f|^{\alpha}$ over a two decade range of positive frequencies, with $B_{1}=5$ and $\alpha=-5 / 3$, and is flat on either side.

\subsection{Theoretical Comparison}

In this section, the theoretical performance of each of the estimators is considered; first the bias, followed by the variance. This effort provides a benchmark for computer simulations and a basis for understanding observed biases in the wavelet variance and structure function estimators.

It is difficult to define a process in terms of its autocovariance sequence (ACVS) since one must ensure that the ACVS is positive semidefinite. For this reason, in Equation 11 we have specified the process in terms of its SDF. In doing so, we have introduced some bias into the estimates of $\alpha$ and $B_{1}$ based upon the structure function and wavelet variance since the mapping of the power law behavior from the SDF is inexact for band-limited processes. The considered process was chosen to have exact power law behavior over a two decade range in its SDF. The wavelet variance and structure function do not display exact power law behavior for this band-limited process; they are nearly linear over a range of scales that is slightly less than two decades in length. The wavelet variance that corresponds to the chosen SDF was obtained through numerical integration of

$$
\nu_{X}^{2}\left(\tau_{j}\right)=\int_{-1 / 2}^{1 / 2}\left|\widetilde{H}_{j}(f)\right|^{2} S_{X}(f) d f
$$

where $\widetilde{H}_{j}$ is the transfer function for the filter $\left\{\tilde{h}_{j, l}\right\}$. The structure function was obtained from

$$
D_{X}(\tau)=2\left(s_{X, 0}-s_{X, \tau}\right)
$$

after computing the ACVS $s_{X, \tau}$ from the inverse discrete Fourier transform of the SDF. Regressions were performed (over identical ranges of scale as in the empirical comparisons) on this wavelet variance and structure function to determine the corresponding values for the estimators of the parameters $\alpha$ and $B_{1}$. The values differ slightly from the ones prescribed in Equation 11. This difference is related to the imperfect mapping of the power law behavior of the simulated process rather than the performance of the individual estimators. Tables 1 and 2 summarize the theoretical performance of the estimators of the parameters $\alpha$ and $B_{1}$. The LA(16) wavelet was selected because it provides a good approximation to a band-pass filter. Since we know the true SDF for 
the process, we are able to estimate the introduced bias, along with the bias that results from the band-pass filtering approximation in the wavelet variance formulation. It seems most appropriate to compare only the magnitude of the variance, of each of the estimators, to avoid this introduced bias.

The theoretical variances for the estimators of the parameter $\Theta=[c, \alpha]^{T}$ in the linear regression model of Equation 2 for the multitaper case are given by the diagonal elements of the covariance matrix shown in Equation 3. Since $c=\log \left(B_{1}\right)+\psi(K)-\log (K)$, the least squares estimator $\hat{c}$ of $c$ can be used to define an estimator for $B_{1}$, namely,

$$
\hat{B}_{1}^{(m t)} \equiv \exp \{\hat{c}-\psi(K)+\log (K)\} .
$$

Noting that

$$
\operatorname{var}\left\{\hat{B}_{1}^{(m t)}\right\}=K^{2} \exp \{-2 \psi(K)\} \operatorname{var}\{\exp (\hat{c})\}
$$

and assuming that $\hat{c}$ is normally distributed, then $\exp (\hat{c})$ obeys a log normal distribution with parameters $\mu=\log \left(B_{1}\right)+\psi(K)-\log (K)$ and $\sigma^{2}$ given by the upper left-hand element of the covariance matrix in Equation 3. A standard result says that the variance of $\exp (\hat{c})$ is given by $\exp \left(2 \mu+\sigma^{2}\right)\left\{\exp \left(\sigma^{2}\right)-1\right\}$, and hence we have

$$
\operatorname{var}\left\{\hat{B}_{1}^{(m t)}\right\}=B_{1}^{2} \exp \left(\sigma^{2}\right)\left(\exp \left(\sigma^{2}\right)-1\right) .
$$

The variance of the least squares estimator $\hat{\alpha}$ of $\alpha$ is given by the lower righthand element of the covariance matrix in Equation 3. In the wavelet variance case, the theoretical variances for the estimator of the parameters $\Theta=[\zeta, \beta]^{T}$ can be obtained from an expression analogous to Equation 3 for the ordinary least squares estimator and from Equation 10 for the weighted least squares estimator. The variance of the wavelet variance estimator of $B_{1}$ is

$$
\operatorname{var}\left\{\hat{B}_{1}^{(w v)}\right\}=\left(\frac{\alpha+1}{2 B_{1}\left(2^{-(\alpha+1)}-4^{-(\alpha+1)}\right)}\right)^{2} e^{2 \mu+\operatorname{var}\left\{\hat{\zeta}^{(w v)}\right\}}\left(e^{\operatorname{var}\left\{\hat{\zeta}^{(w v)}\right\}}-1\right),
$$

where $\mu$ is the actual mean $\log \left(\frac{2 B_{1}\left(2^{-(\alpha+1)}-4^{-(\alpha+1)}\right)}{\alpha+1}\right)$ and $\alpha=-\frac{5}{3}$ is the actual value of $\alpha$. The theoretical variances for the wavelet variance estimators of $\alpha$ and $\beta$ are the same.

\subsection{Empirical Comparison}

Realizations of the process described by Equation 11 were generated using the Gaussian spectral synthesis method (GSSM) [15]. GSSM is an approximate frequency domain method that can be used to simulate a zero mean Gaussian stationary process with a specified SDF. A segment of length $N$ is sampled 
Table 1

Theoretical performance of $\alpha$ estimators.

\begin{tabular}{|l|c|c|c|c|c|}
\hline & $\alpha$ & bias & bias $^{2}$ & variance & MSE \\
\hline$\hat{S}_{X}^{(m t)}$ & -1.666667 & 0.000000 & 0.000000 & 0.000829 & 0.000829 \\
\hline$\hat{D}_{X}[1,100]$ & -1.701854 & -0.035187 & 0.001238 & no theory & no theory \\
\hline$\hat{D}_{X}[1,64]$ & -1.674131 & -0.007464 & 0.000076 & no theory & no theory \\
\hline$\hat{D}_{X}[2,100]$ & -1.707376 & -0.040709 & 0.001657 & no theory & no theory \\
\hline$\hat{D}_{X}[2,64]$ & -1.677027 & -0.010360 & 0.000107 & no theory & no theory \\
\hline$\hat{\nu}_{X}^{2}$ OLS & -1.669780 & -0.003113 & 0.000010 & 0.002131 & 0.002140 \\
\hline$\hat{\nu}_{X}^{2}$ WLS & -1.664584 & 0.002083 & 0.000004 & 0.000800 & 0.000804 \\
\hline
\end{tabular}

Table 2

Theoretical performance of $B_{1}$ estimators.

\begin{tabular}{|l|c|c|c|c|c|}
\hline & $B_{1}$ & bias & bias $^{2}$ & variance & MSE \\
\hline$\hat{S}_{X}^{(m t)}$ & 5.000000 & 0.000000 & 0.000000 & 0.130876 & 0.130876 \\
\hline$\hat{D}_{X}[1,100]$ & 5.105634 & 0.105634 & 0.011159 & no theory & no theory \\
\hline$\hat{D}_{X}[1,64]$ & 4.753328 & -0.246672 & 0.060847 & no theory & no theory \\
\hline$\hat{D}_{X}[2,100]$ & 5.215992 & 0.215992 & 0.046652 & no theory & no theory \\
\hline$\hat{D}_{X}[2,64]$ & 4.801153 & -0.198847 & 0.039540 & no theory & no theory \\
\hline$\hat{\nu}_{X}^{2}$ OLS & 5.076332 & 0.076332 & 0.005827 & 0.154553 & 0.160380 \\
\hline$\hat{\nu}_{X}^{2}$ WLS & 5.122484 & 0.122484 & 0.015002 & 0.046261 & 0.061263 \\
\hline
\end{tabular}

from a harmonic process of length $M$ that is generated using a DFT approach. Percival [15] shows that, by making $M$ large, the ACVS of the simulated series can be made arbitrarily close to the desired ACVS out to lag $N-1$. We experimented with different values of $M$ to determine their effect on the structure function. Since the structure function may be expressed in terms of the ACVS (Equation 13), the structure function of the simulated process will also converge to the desired structure function as $M$ becomes large. Estimates of $\alpha$ and $B_{1}$ were obtained using the structure function for 10,000 realizations of the simulated process with $M=4 N, M=8 N, M=16 N$, and $M=32 N$. No significant change in the estimates of $\alpha$ or $B_{1}$ was observed with increasing $M$, suggesting that $M=4 N$ is large enough to produce accurate results.

Estimates of $B_{1}$ and $\alpha$ were obtained with each of the estimators for 10,000 realizations of the simulated process. The multitaper method used $K=5$ sine tapers, and the regression was performed on frequencies in the power-law portion of the SDF. The structure function was calculated over four different ranges of unitless separations: 1 to 100,2 to 100,1 to 64 , and 2 to 64 . Using 
Table 3

Summary of $\alpha$ estimates.

\begin{tabular}{|l|c|c|c|c|c|}
\hline & $\hat{\alpha}$ & bias & bias $^{2}$ & variance & MSE \\
\hline$\hat{S}_{X}^{(m t)}$ & -1.666779 & -0.000112 & 0.000000 & 0.000717 & 0.000717 \\
\hline$\hat{D}_{X}[1,100]$ & -1.703846 & -0.037179 & 0.001382 & 0.001160 & 0.002542 \\
\hline$\hat{D}_{X}[1,64]$ & -1.675542 & -0.008875 & 0.000079 & 0.000814 & 0.000893 \\
\hline$\hat{D}_{X}[2,100]$ & -1.709559 & -0.042892 & 0.001840 & 0.001404 & 0.003244 \\
\hline$\hat{D}_{X}[2,64]$ & -1.678617 & -0.011950 & 0.000143 & 0.001033 & 0.001176 \\
\hline$\hat{\nu}_{X}^{2}$ OLS & -1.664909 & 0.001758 & 0.000003 & 0.001848 & 0.001851 \\
\hline$\hat{\nu}_{X}^{2}$ WLS & -1.662119 & 0.004548 & 0.000021 & 0.000746 & 0.000767 \\
\hline
\end{tabular}

Table 4

Summary of $B_{1}$ estimates.

\begin{tabular}{|l|c|c|c|c|c|}
\hline & $\hat{B}_{1}$ & bias & bias $^{2}$ & variance & MSE \\
\hline$\hat{S}_{X}^{(m t)}$ & 5.011043 & 0.011043 & 0.000122 & 0.113948 & 0.114070 \\
\hline$\hat{D}_{X}[1,100]$ & 5.140074 & 0.140074 & 0.019621 & 0.147397 & 0.167018 \\
\hline$\hat{D}_{X}[1,64]$ & 4.771829 & -0.228171 & 0.052062 & 0.065295 & 0.117358 \\
\hline$\hat{D}_{X}[2,100]$ & 5.261158 & 0.261158 & 0.068203 & 0.219234 & 0.287438 \\
\hline$\hat{D}_{X}[2,64]$ & 4.826778 & -0.173222 & 0.030006 & 0.105196 & 0.135202 \\
\hline$\hat{\nu}_{X}^{2}$ OLS & 5.122879 & 0.122879 & 0.015099 & 0.138459 & 0.153558 \\
\hline$\hat{\nu}_{X}^{2}$ WLS & 5.137826 & 0.137826 & 0.018996 & 0.043069 & 0.062065 \\
\hline
\end{tabular}

Daubechies LA(16) wavelets, ordinary and weighted least squares estimates were obtained on wavelet transform levels $j=2$ through $j=7$ using the unbiased estimate of the wavelet variance. The squared bias, variance, and mean squared error (MSE) of each of the estimators are summarized in Table 3 for $\alpha$, Table 4 for $B_{1}$, and Table 5 for estimates of $B_{1}$ obtained through scaling range averaging. Figures 2 through 4 show corresponding box and whisker diagrams. For $\alpha$ (Table 3 and Figure 2), the multitaper and weighted least squares wavelet estimates have about $10 \%$ less variance than the best structure function estimate, which is based on $[1,64]$. For $B_{1}$ estimated via $\hat{B}_{1}$ (Table 4 and Figure 3$)$, the structure function $[1,64]$ and $[2,64]$ estimates have smaller variances than the multitaper estimate, but the weighted least squares wavelet estimate has the smallest variance. For $B_{1}$ estimated based upon scaling range averaging (Table 5 and Figure 4), all of the structure functions estimates are better than the multitaper and wavelet variance estimates in terms of variance.

A comparison of Tables 3 and 4 with Tables 1 and 2 indicates very good agreement between theoretical and corresponding empirical values for all es- 
Table 5

Summary of estimates of $B_{1}$ obtained using scaling range averaging.

\begin{tabular}{|l|c|c|c|c|c|}
\hline & $\hat{B}_{1}$ & bias & bias $^{2}$ & variance & MSE \\
\hline$\hat{S}_{X}^{(m t)}$ & 5.010497 & 0.010479 & 0.000110 & 0.064256 & 0.064366 \\
\hline$\hat{D}_{X}[1,100]$ & 4.575704 & -0.424296 & 0.180028 & 0.024661 & 0.204689 \\
\hline$\hat{D}_{X}[1,64]$ & 4.612862 & -0.387138 & 0.149876 & 0.019428 & 0.169304 \\
\hline$\hat{D}_{X}[2,100]$ & 4.601591 & -0.398401 & 0.158729 & 0.032927 & 0.191656 \\
\hline$\hat{D}_{X}[2,64]$ & 4.649701 & -0.350299 & 0.122709 & 0.026435 & 0.149144 \\
\hline$\hat{\nu}_{X}^{2}$ & 5.091149 & 0.091149 & 0.008308 & 0.045419 & 0.053727 \\
\hline
\end{tabular}

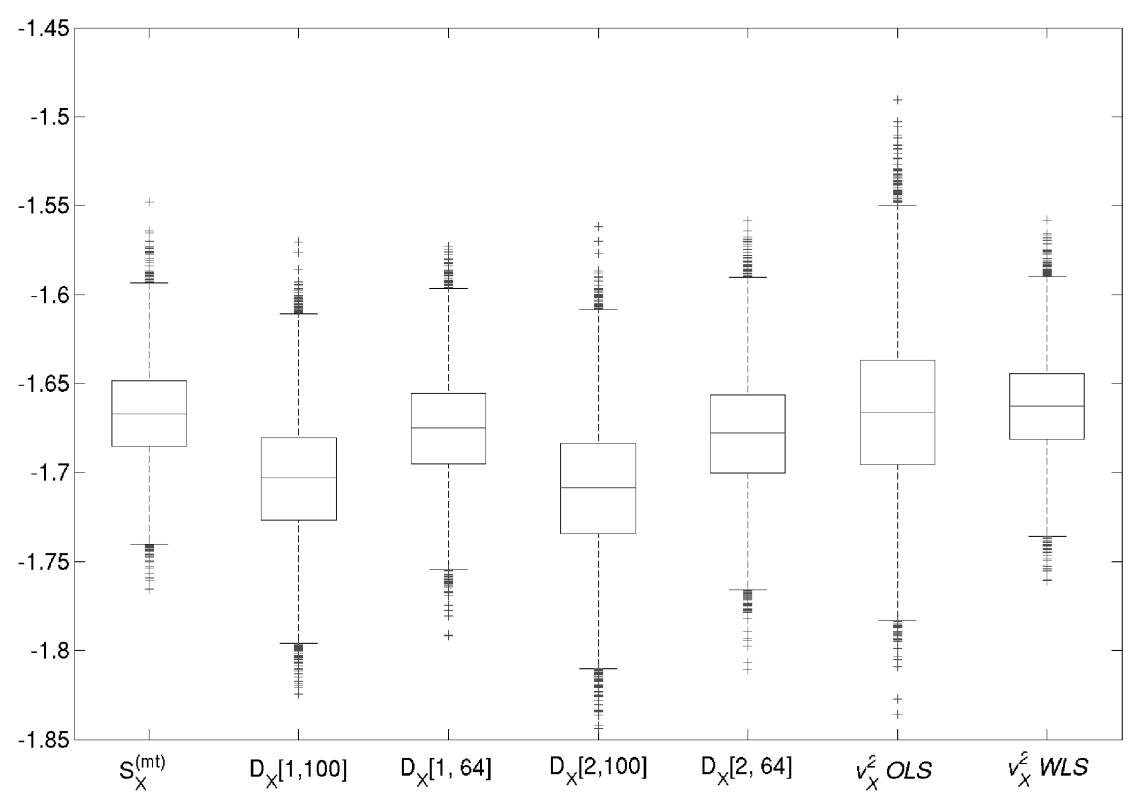

Fig. 2. Box and whisker diagram comparison of $\alpha$ estimates.

timators. In particular, the empirical estimates of $\alpha$ and $B_{1}$ agree well with the theoretical values for each estimator. The theoretical predictions of the variance of the multitaper and wavelet variance estimators of $\alpha$ and $B_{1}$ are between $7 \%$ and $16 \%$ larger than observed, indicating that the sampling theory provides accurate, but somewhat conservative, predictions of the variability of the estimate.

\section{Analysis of Measured Turbulence Data}

Here we use an aerothermal time series to demonstrate the practical use of the multitaper spectral, wavelet and structure function estimators of $\alpha$ and 


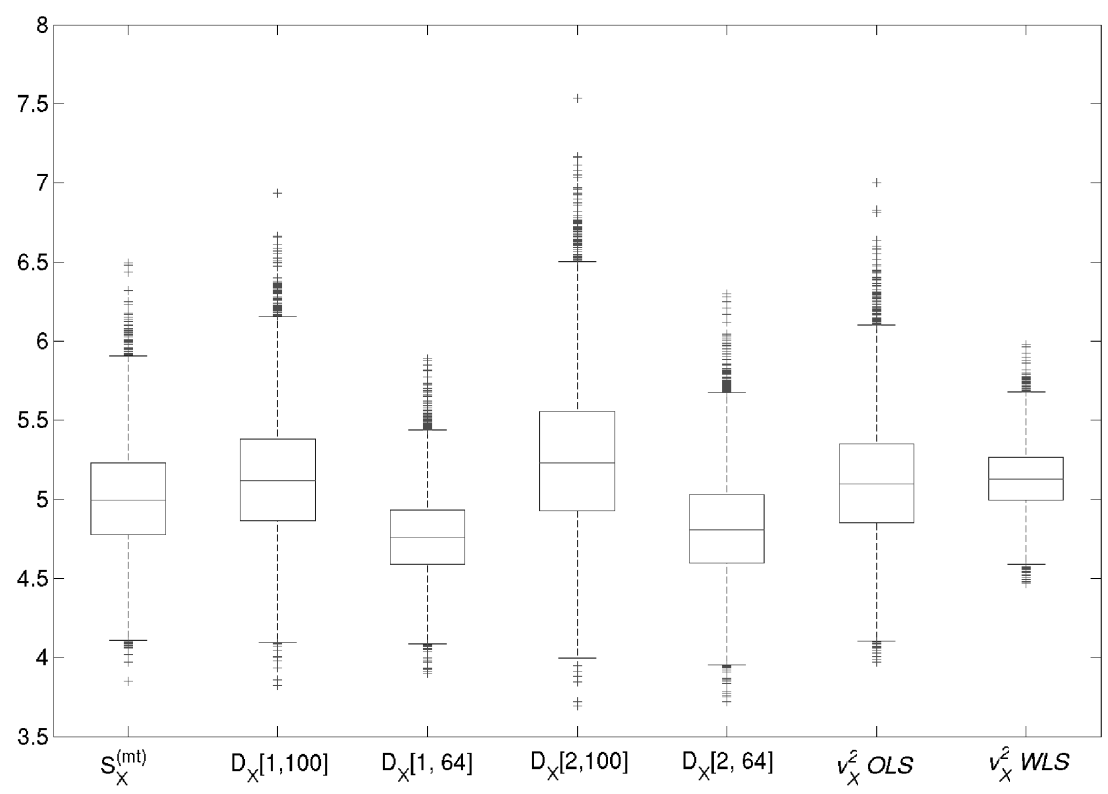

Fig. 3. Box and whisker diagram comparison of $B_{1}$ estimates.

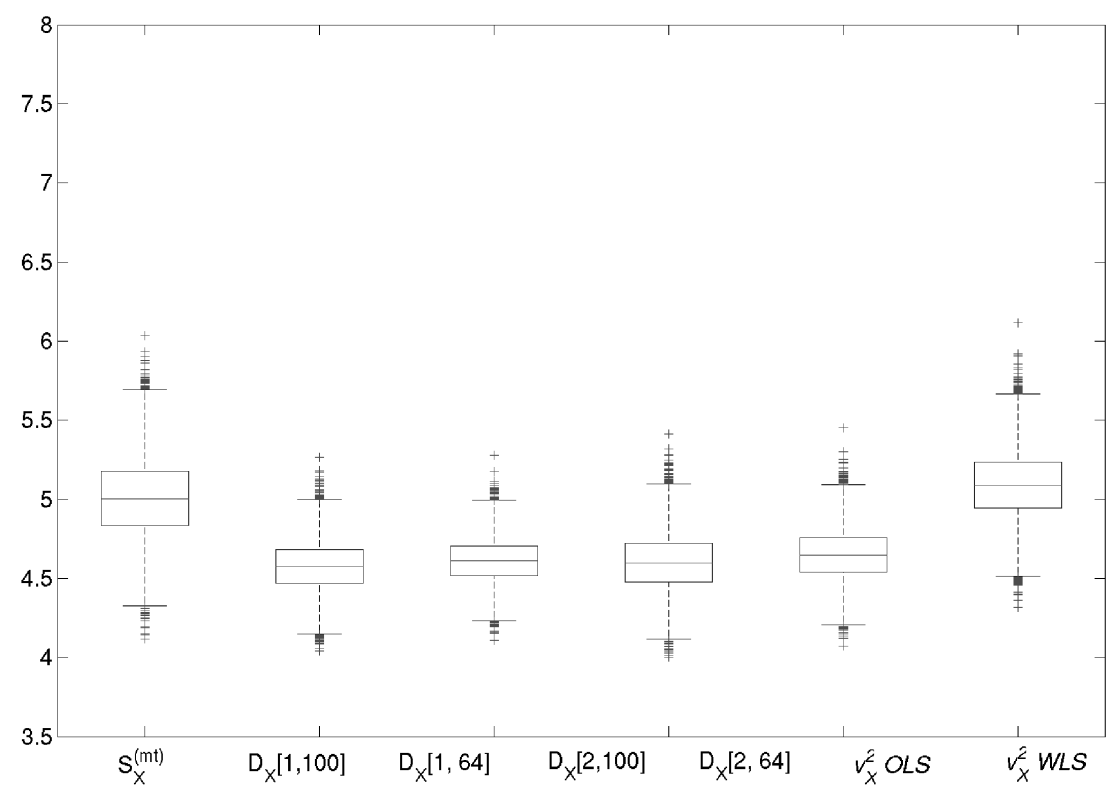

Fig. 4. Box and whisker diagram comparison of estimates of $B_{1}$ obtained through scaling range averaging.

$B_{1}$. The aerothermal series (Figure 5) was recorded using a cold wire probe mounted on an aircraft that flew at a constant or linearly increasing altitude during clear air conditions. The series was uniformly sampled at an average spacing of $1.83 \mathrm{~cm}$ along a flight segment that was $137.3 \mathrm{~km}$ in length. 
It exhibits inhomogeneities that might indicate regions with different turbulent characteristics. Two prominent bursts (regions of high-frequency, highamplitude activity) are evident: one between 20 and $30 \mathrm{~km}$ and the second between 70 and $80 \mathrm{~km}$. The character of the series beyond the second bursting region is quite different from its character between the two bursting regions in that it appears to lack the small-scale variability present earlier on. This is consistent with a conceptual image of an aircraft flying horizontally through a tilted turbulent layer or climbing slowly through a horizontal turbulent layer that is mechanically turbulent throughout with large temperature gradients, caused by mixing, at the layer top and bottom [6]. Mechanical turbulence in the presence of these large temperature gradients could cause the large fluctuations in temperature that are observed in the bursting regions.

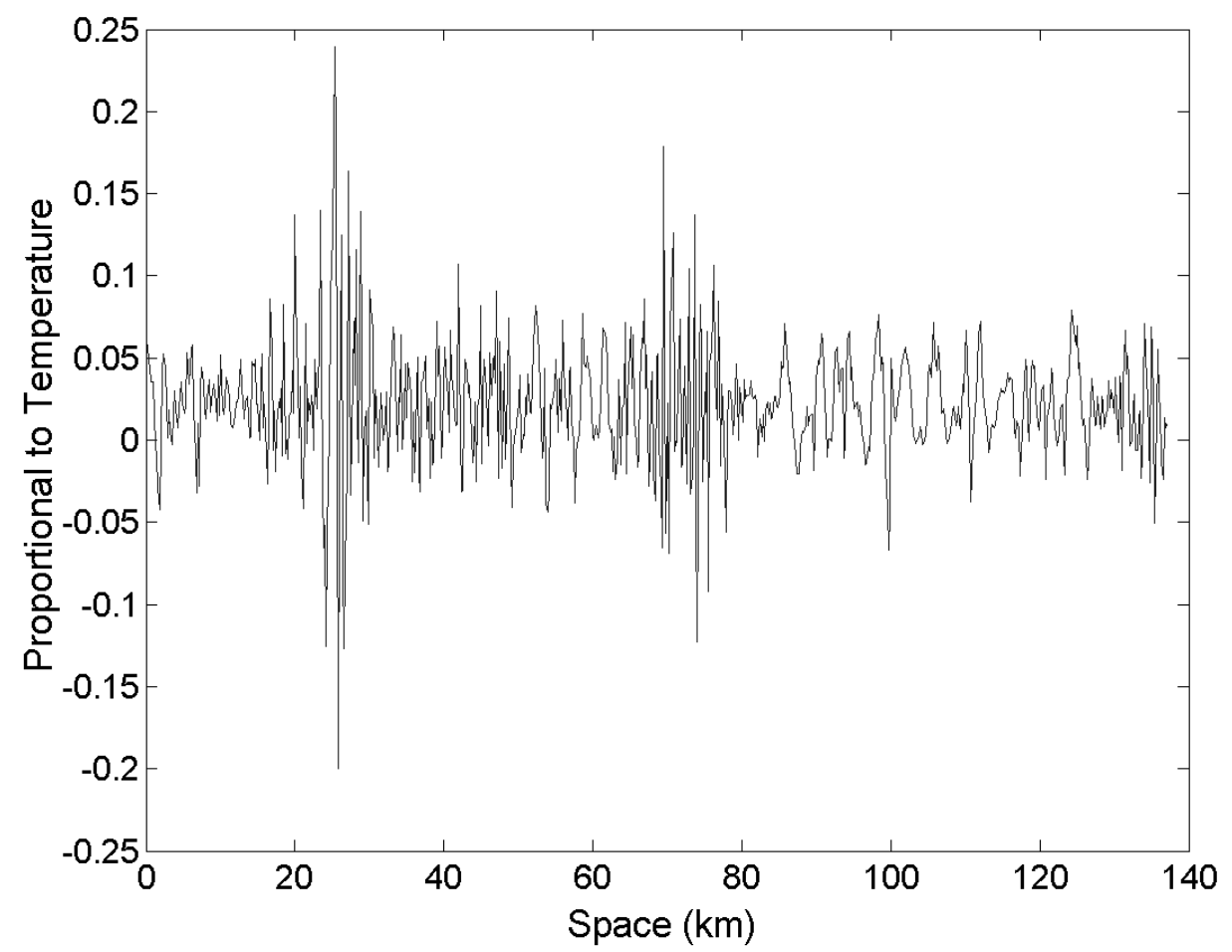

Fig. 5. The aerothermal series.

Constantine et al. [4] previously showed that, to a good approximation, the aerothermal series can be modeled as a time-varying fractionally differenced (FD) process and used an FD process model and discrete wavelet transforms to produce instantaneous estimates of the power law exponent. Their results indicate that the power law exponent within and between the bursting regions is approximately consistent with KOC type behavior for physical scales between $0.59 \mathrm{~m}$ and $18.77 \mathrm{~m}$ with a significant departure from this behavior in the regions prior to the first, and subsequent to the second, bursting regions. They also noted a quasi-periodic contamination of the signal at physical scales 0.1465 and $0.2930 \mathrm{~m}$ which may be due to an acoustic jet screech 
interaction $[9]$.

Because the aerothermal series exhibits characteristics that vary spatially, shorter blocks of data were analyzed in an attempt to ensure that each segment was approximately statistically homogeneous. The smallest wavelet scale apparently unaffected by the quasi-periodic contamination is $j=6$, which corresponds to a physical scale of $0.59 \mathrm{~m}$ [4]. The weighted least squares wavelet variance estimates of $\alpha$ and $B_{1}$ were computed using wavelet scales $j=6$ through $j=8$ and the $\mathrm{LA}(16)$ wavelet. This corresponds to a frequency band $0.1067<f \leq 0.8533 \mathrm{~m}^{-1}$. The length, $N$, of these segments was chosen to prevent bias in the multitaper estimates by ensuring that the smallest Fourier ordinate in the regression is farther from the zero-frequency component than the half-width of the multitaper spectral bandwidth, $W^{\prime} \equiv$ $(K+1) /\{2(N+1) \Delta x\}[12]$, where the number of data tapers was chosen to be $K=5$. Segments of length $N=2^{14}$ were chosen, each representing a distance of approximately $0.3 \mathrm{~km}$ along the flight path. The structure function estimates were computed using a range of separation distances between 0.59 and $2.34 \mathrm{~m}$. The estimates of $\alpha$ and $B_{1}$ obtained through these procedures are displayed in Figures 6 and 7 . The estimates of $B_{1}$ were all obtained using the scaling range averaging procedure.

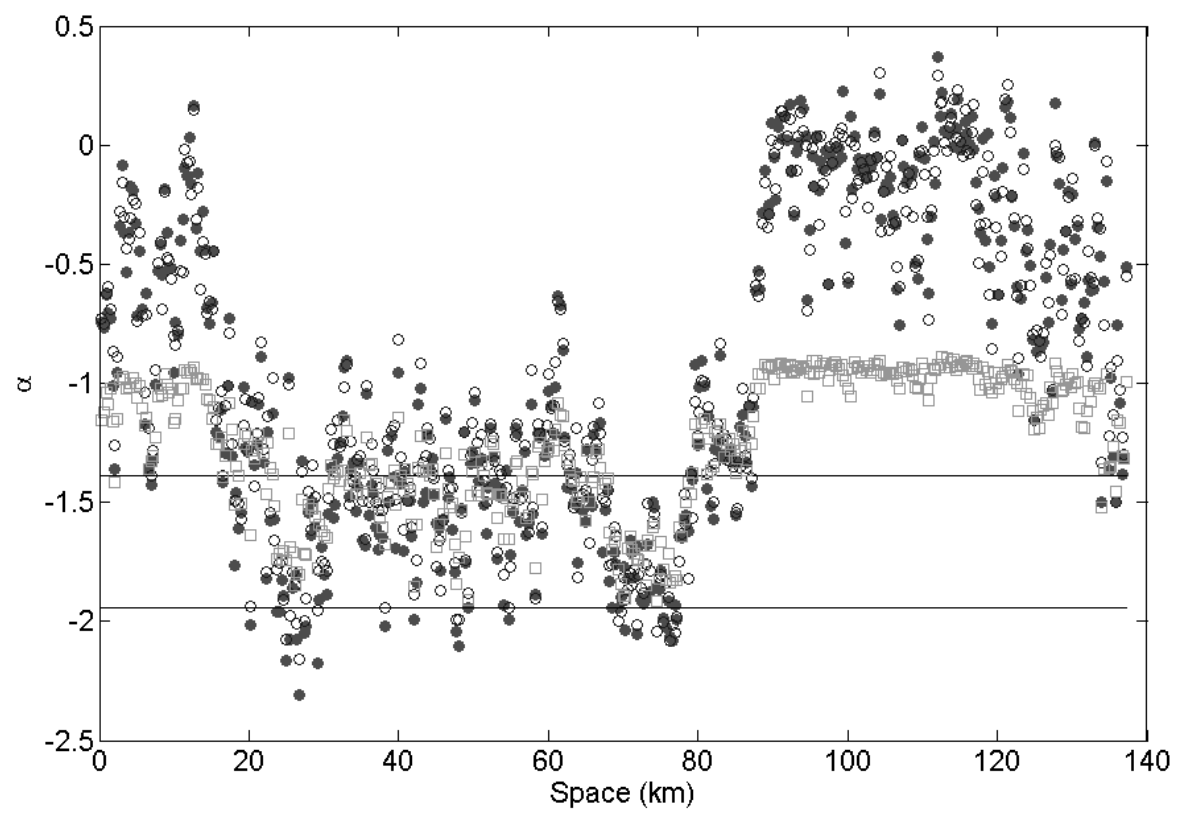

Fig. 6. Multitaper spectral (open circles), wavelet variance (dots), and structure function (open squares) estimates of $\alpha$ for length $N=2^{14}$ blocks of the aerothermal series. The two horizontal solid lines represent the KOC value $(-5 / 3) \pm 95 \%$ confidence intervals from the multitaper spectral estimator.

In agreement with Constantine et al. [4], the multitaper spectral and wavelet variance estimates of the power law exponent $\alpha$ (Figure 6) are near the KOC 


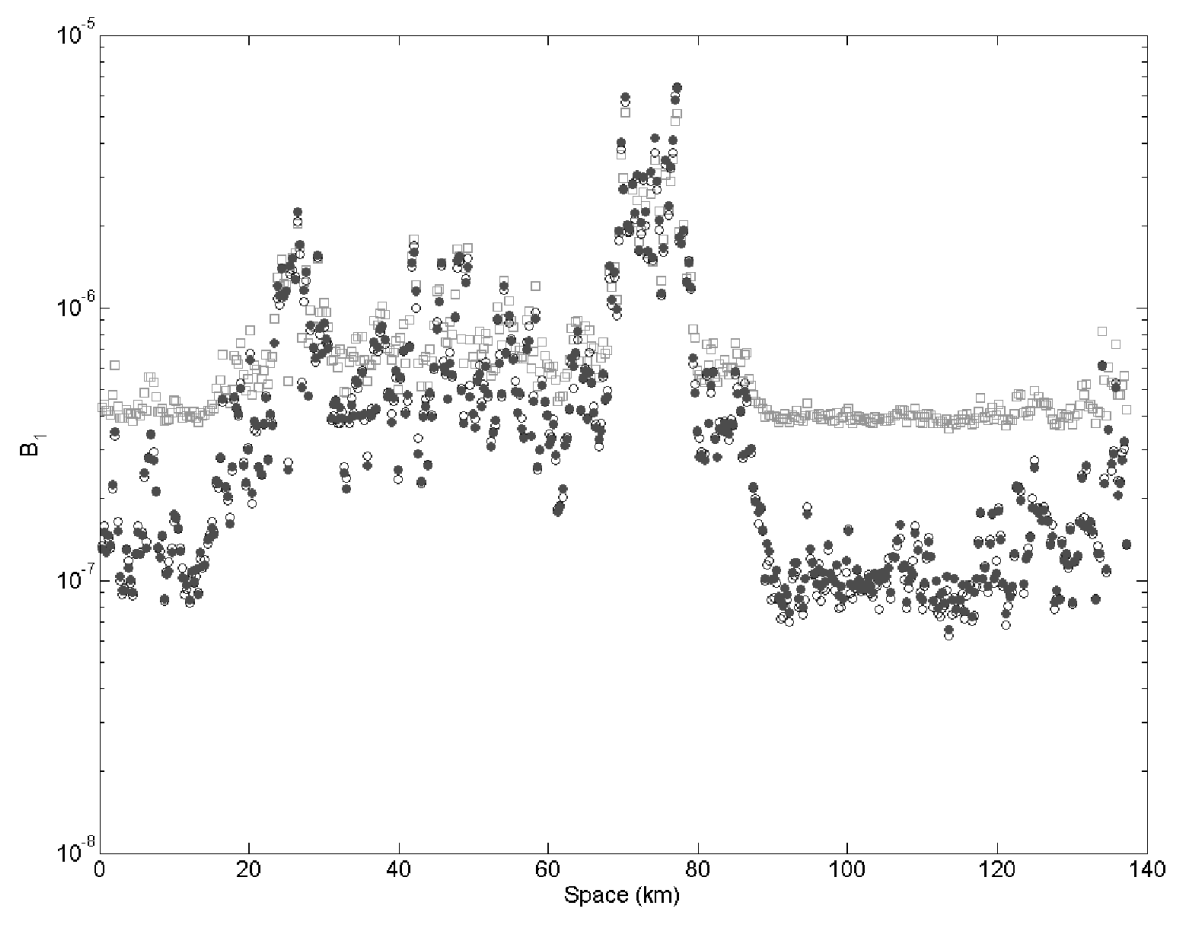

Fig. 7. Scaling range averaged multitaper spectral (open circles), wavelet variance (dots), and structure function (open squares) estimates of $B_{1}$ for length $N=2^{14}$ blocks of the aerothermal series. The y-axis is dimensionless because the series is uncalibrated.

$-5 / 3$ value between approximately 20 and $80 \mathrm{~km}$ and take on values that are significantly smaller in magnitude for much of the remainder of the series. The structure function estimates of $\alpha$ are also near the KOC value between 20 and $80 \mathrm{~km}$, but, in comparison to the other estimates, take on values that are larger in magnitude elsewhere. This disagreement is expected since the structure function estimator of $\alpha$ is only valid for $-3 \leq \alpha<-1$. Note that many of the estimates from the region between 20 and $80 \mathrm{~km}$ fall outside the confidence intervals shown in Figure 6. We would expect roughly $95 \%$ of these estimates to be within these limits if a $-5 / 3$ power law were obeyed throughout; a possible interpretation is that KOC type behavior is only observed intermittently.

The estimates of the constant of proportionality are significantly higher in the region between 20 and $80 \mathrm{~km}$ than they are elsewhere (Figure 7). The structure function estimates of $B_{1}$ are significantly different in the regions where $\alpha$ deviates from $-5 / 3$ because, again, the structure function estimator is only valid for $-3 \leq \alpha<-1$. Note that the $B_{1}$ estimates were obtained using the scaling range averaging procedure and, as a result, are proportional to the energy content within the chosen scaling range and not directly related to the $y$-intercept of the regression line. 
A comparison of the estimates of $\alpha$ (Figure 6) and $B_{1}$ (Figure 7 ) would seem to indicate a link between KOC behavior and the strength of the temperature fluctuations since the estimates for $B_{1}$ are largest in the regions where $\alpha$ is near $-5 / 3$; however, it is possible that the temperature fluctuations in portions of the time series are too small to be resolved by the cold wire probe, resulting in minimal values for $B_{1}$ and meaningless estimates of $\alpha$. This possibility could not be tested because calibration and sensitivity data for the temperature probe were not available.

Longer segments of the aerothermal data were analyzed to explore the behavior of fluctuations with larger physical scales. These segments were of length $N=$ $2^{20}$, each representing a distance of approximately $19.2 \mathrm{~km}$ along the flight path. Figures 8 and 9 show the resulting estimates for $\alpha$ and $B_{1}$. The regression for the wavelet variance estimators were performed over wavelet scales $j=6$ through $j=12$, corresponding to physical scales between 0.59 and $37.5 \mathrm{~m}$. The regression for the multitaper spectral estimators spanned a frequency band $0.006667<f \leq 0.8533 \mathrm{~m}^{-1}$. The structure function estimates were based on separation distances between 0.59 and $37.5 \mathrm{~m}$. The estimates of $\alpha$ and $B_{1}$ from these longer segments are both qualitatively and quantitatively similar to those using length $N=2^{14}$ (Figures 6 and 7). In both cases, the $\alpha$ estimates are near the KOC value of $-5 / 3$ in the region between 20 and $80 \mathrm{~km}$ and deviate from this value elsewhere; the largest estimates for $B_{1}$ are also observed in this region. This suggests that the same power law behavior that was witnessed at smaller scales (Figures 6 and 7) extends to the larger scales as well.

To this point we have merely assumed that the aerothermal series obeys a power law within each segment that we have analyzed. This assumption may be tested by looking at the residuals: $Y^{\prime(m t)}(f)=Y_{j}-\hat{Y}_{j}$ for the multitaper estimate of the SDF, $Y^{\prime(s f)}(\tau)=Y_{\tau}-\hat{Y}_{\tau}$ for the structure function, and $Y^{\prime(w v)}\left(\tau_{j}\right)=Y\left(\tau_{j}\right)-\hat{Y}\left(\tau_{j}\right)$ for the wavelet variance, where $\hat{Y}_{X}$ denotes the estimate of $Y_{X}$ obtained by performing the regression on the appropriate characterization. Figure 10 displays the residuals for the multitaper estimate of the SDF for the first and second segments of length $N=2^{20}$ and the $95 \%$ confidence intervals appropriate for a $\log \chi_{2 K}^{2}$ random variable with $K=5$ data tapers. While the vast majority of the points fall within the approximate $95 \%$ confidence intervals, the residuals for the first segment display a trend that indicates that the linear approximation is not adequate to describe the SDF over the scaling range. The residuals for the second segment do not display this trend, indicating that the linear approximation provides a much more suitable description of the SDF here. The linear approximation seems appropriate for the segments with $\alpha$ estimates in the $-5 / 3$ region and less adequate for the remainder of the series. Figure 11 shows the residuals and $95 \%$ confidence intervals for the regression on the wavelet variance estimate. The residuals for the second segment all fall within the confidence intervals 


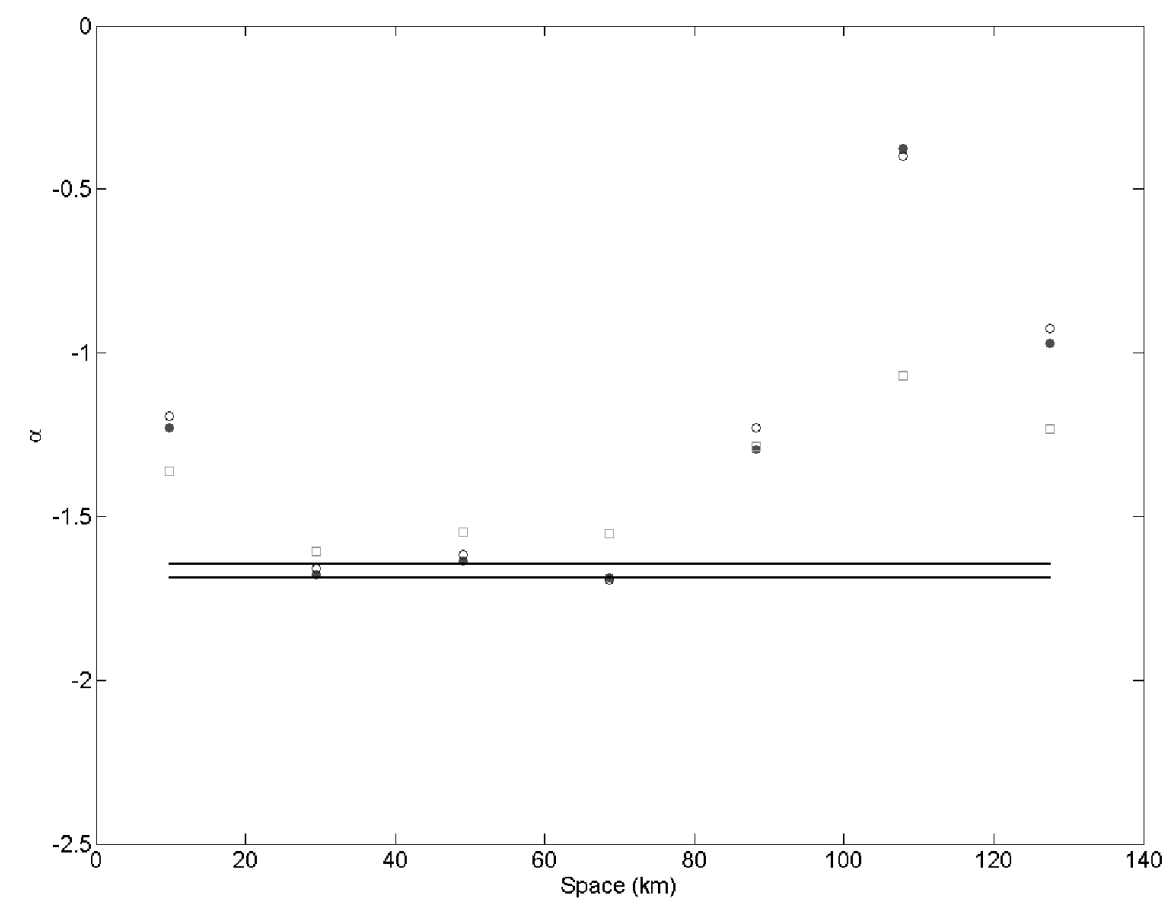

Fig. 8. Multitaper spectral (open circles), wavelet variance (dots), and structure function (open squares) estimates of $\alpha$ for length $N=2^{20}$ blocks of the aerothermal series. The two horizontal solid lines represent the KOC value $(-5 / 3) \pm 95 \%$ confidence intervals from the multitaper spectral estimator.

and lack any obvious trend which would indicate a deficiency in the linear model; the linear approximation is clearly inadequate for the first segment. The linear approximation provides an adequate description of the SDF and wavelet variance for the second, third and fourth segments of the aerothermal series, i.e., the segments within and between the bursting regions that yield the $\alpha$ values nearest to $-5 / 3$ and the largest values for $B_{1}$.

While the residuals for the multitaper and wavelet estimates allow us to assess the adequacy of the power law assumption, the same cannot be said for the structure function. Figure 12 shows the structure function residuals for the same two segments considered in Figures 10 and 11. No confidence intervals are available for these plots because no useful internal error estimates are available for the structure function. The oscillatory behavior apparent in the residuals results from the quasi-periodic noise that is confined to small scales in the wavelet variance and SDF, but unfortunately contaminates the entire range of separation distances in the structure function. This is a shortcoming of the structure function in analyzing any type of signal with harmonic or quasiperiodic components. The structure function residuals from the aerothermal data are highly correlated and display obvious trends, in addition to the oscillations, indicating that the linear approximation is a poor descriptor of the 


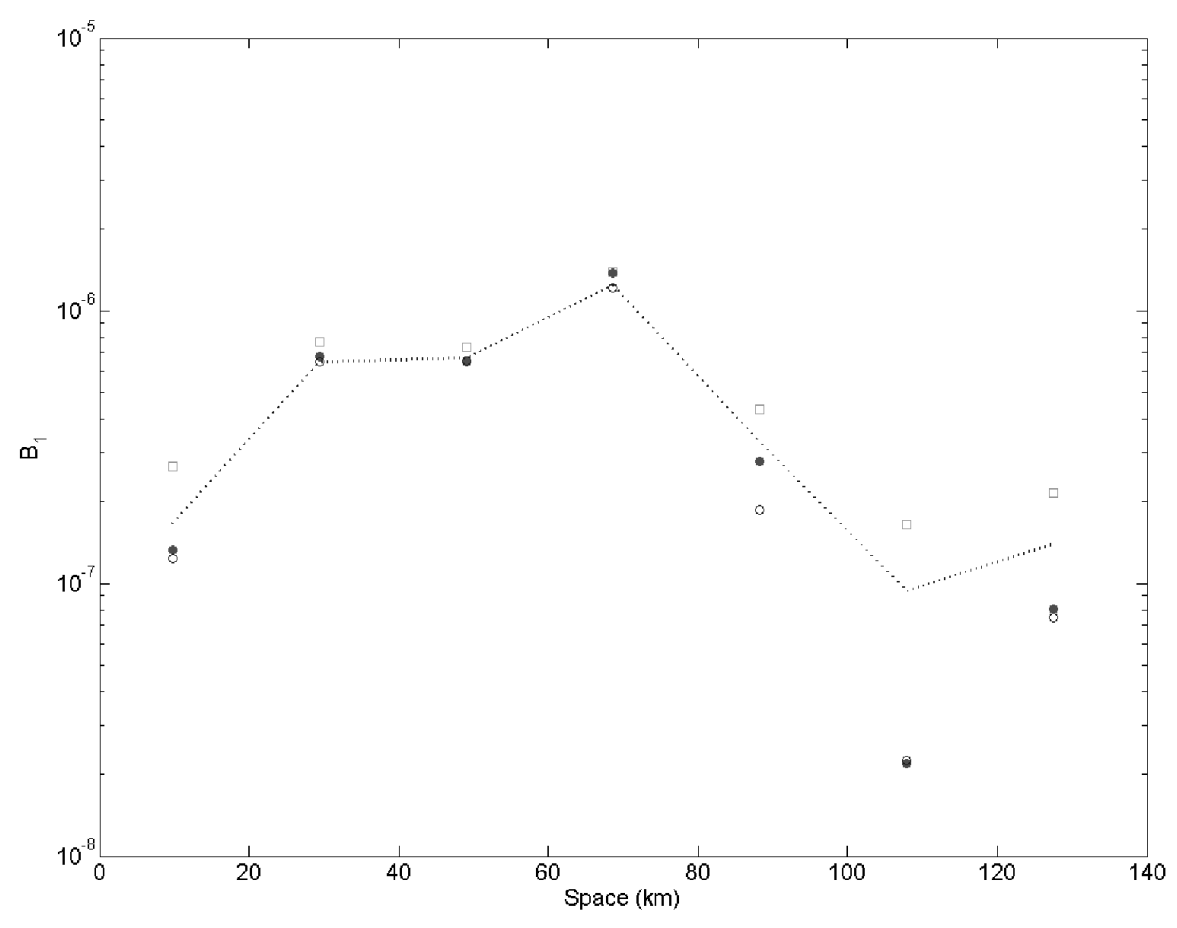

Fig. 9. Scaling range averaged multitaper spectral (open circles), wavelet variance (dots), and structure function (open squares) estimates of $B_{1}$ for length $N=2^{20}$ blocks of the aerothermal series. The dotted line represents the estimates of $B_{1}$ obtained using the multitaper spectral estimator with the Fourier frequency ordinates limited to $\frac{1}{512}<f_{j} \leq \frac{1}{64}$. The y-axis is dimensionless because the aerothermal series is uncalibrated.
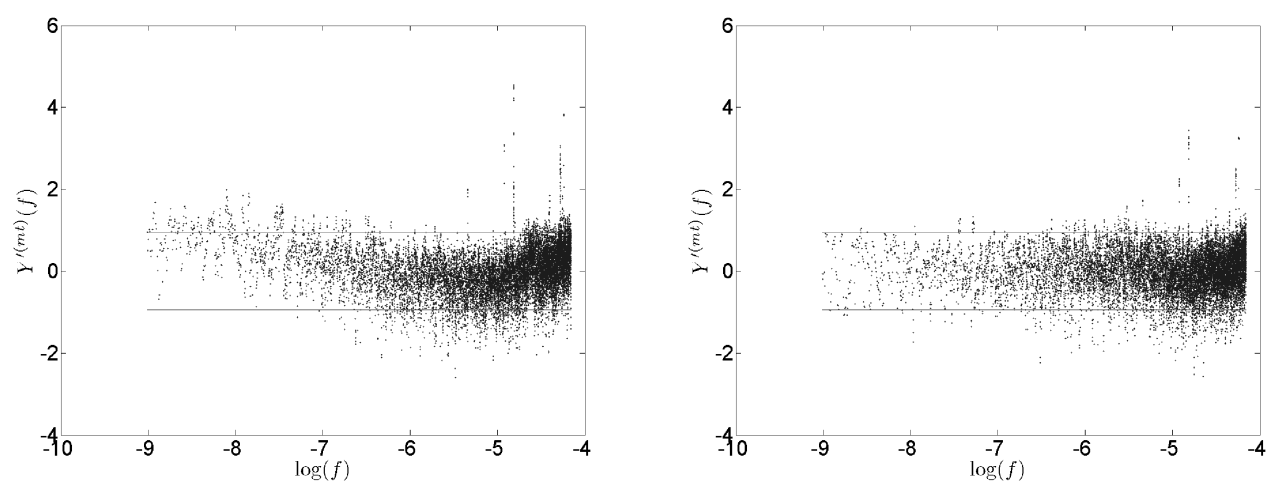

Fig. 10. Regression residuals for the multitaper spectral estimator applied to the aerothermal series. The left hand panel refers to the first segment of length $N=2^{20}$, the right hand panel refers to the second segment. The solid lines indicate approximate $95 \%$ confidence intervals.

structure function. The SDF and wavelet variance are superior characterizations for this turbulent time series because they isolate the quasi-periodic noise at high frequencies and small scales and, more importantly, because they are 

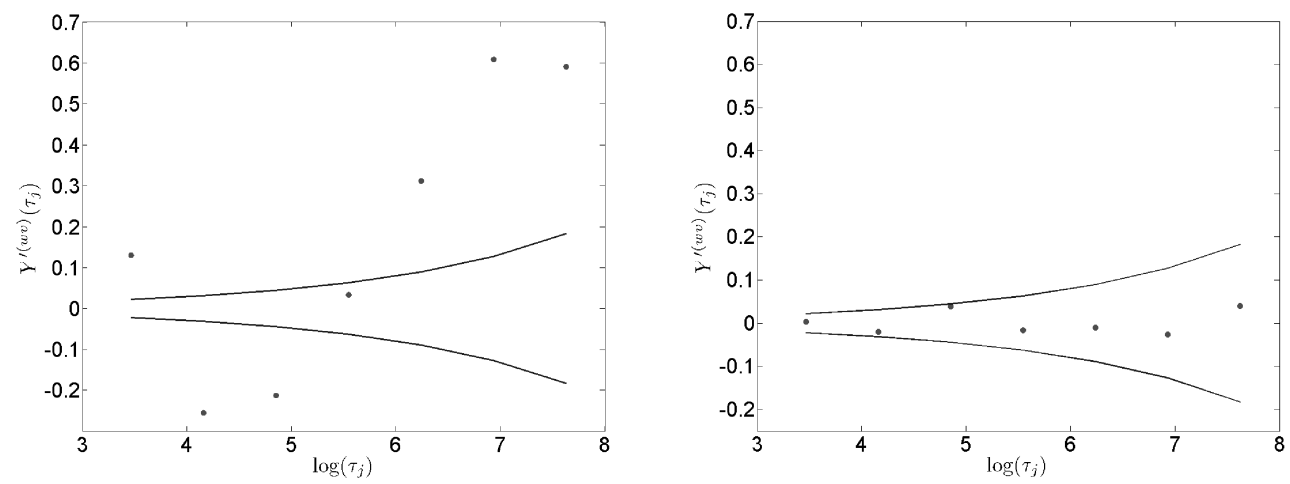

Fig. 11. Regression residuals for the wavelet variance estimator applied to the aerothermal series. The left hand panel refers to the first segment of length $N=2^{20}$, the right hand panel refers to the second segment. The solid curves indicate approximate $95 \%$ confidence intervals.

approximately linear over the scaling range, while the structure function is not. There is reason to believe that other turbulence data sets exhibit this same characteristic, since other investigators have noted a more extensive scaling region in the spectral approach than the structure function [3].
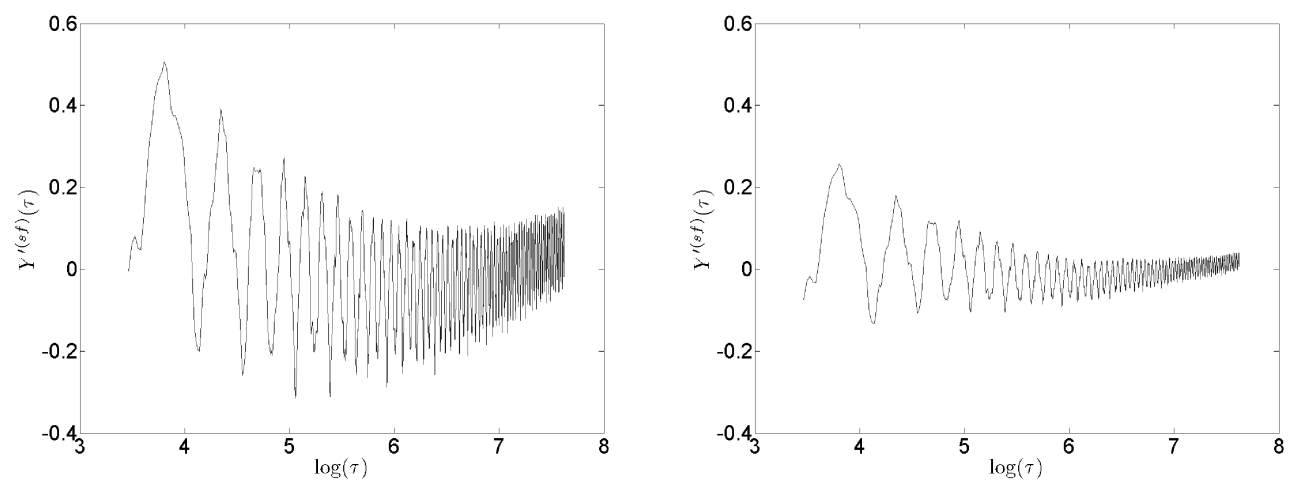

Fig. 12. Regression residuals for the structure function estimator applied to the aerothermal series. The left hand panel refers to the first segment of length $N=2^{20}$, the right hand panel refers to the second segment.

\subsection{Conclusions}

The uncalibrated aerothermal series displays spatially extensive - but not ubiquitous - power law behavior that is approximately consistent with KOC turbulence. This type of turbulence occurs primarily in regions with high levels of activity, and less so in inactive regions; however, we cannot rule out the possibility that this pattern is an artifact of noise due to instrumentation.

This analysis demonstrates the inferiority of the structure function in characterizing the power law behavior of this time series. There is no suitable 
theory available to provide internal error estimates for the structure function, which severely limits our ability to use the residuals to assess the adequacy of the power law assumption. The residuals from the structure function estimate are obviously contaminated by a quasi-periodic component, which, in contrast, was isolated outside of the scaling region by the multitaper spectral and wavelet variance estimates. The structure function residuals are highly correlated and indicate that the structure function is not linear at the same scales that are well characterized by a linear approximation to the SDF and wavelet variance. The SDF and wavelet variance provide simple characterizations for this data set with known statistical properties; the same cannot be said for the structure function.

\section{Discussion}

It has been noted in the turbulence literature (see Antonia and Smalley [3], and references therein) that spectra of turbulent signals sometimes display more extensive scaling regions than do structure function estimates of the same signals. In Section 3 a stationary Gaussian process was defined in terms of its SDF with two decades of power law behavior. The corresponding structure function for this process does not display this power law behavior exactly, but instead shows a much narrower range of scales where the slope is nearly linear. Regressions performed over this nearly linear region yielded estimates of the power law exponent and constant of proportionality that are approximately in agreement with the prescribed values. One can also imagine processes that display more extensive power law behavior in their structure functions than in their SDFs, but these processes are difficult to define because one must ensure that their autocovariance sequences are positive semidefinite. In either case, the process will be more simply described by the characterization displaying the most extensive scaling region; in practice, there is evidence to suggest that this would be the spectral representation.

Multitaper and wavelet methods did better than the structure function approach in estimating $\alpha$ in terms of variance (see Table 3 ) while the wavelet method displayed a smaller variance in estimating $B_{1}$ from the regression intercept than the structure function (see Table 4). More importantly, the variance and MSE can be computed theoretically for the multitaper and wavelet methods, but not for the structure function method, which is a serious limitation in practical applications. The multitaper estimator, the weighted least squares estimates using the LA(16) wavelet, and structure function [1, 64] estimator have the smallest variances for the estimation of $\alpha$. The multitaper, structure function [1, 64], structure function [2, 64], and weighted least squares wavelet estimates have the smallest variances for the estimation of $B_{1}$ from the regression intercept. The variance of the multitaper estimator is $12 \%$ smaller 
than the structure function $[1,64]$ estimator for $\alpha$ and $75 \%$ larger for $B_{1}$. The wavelet variance estimator gives an improvement over the structure function $[1,64]$ of $8 \%$ for $\alpha$ and $34 \%$ for $B_{1}$. Theoretical confidence intervals can be based upon the multitaper spectral estimates and wavelet variance estimates; however, no theory is available to provide confidence intervals based upon the structure function estimates because of their highly correlated nature. The scaling range averaging approach is recommended, in contrast to the regression intercept based estimates, because it provides an estimate of $B_{1}$ that may be interpreted in terms of the signal energy contained in the scaling range, regardless of the slope. The simulations indicate that the best estimates of $B_{1}$ are also obtained through scaling range averaging. Scaling range averaging produces estimates with variances that are very similar to the weighted least squares estimator for the wavelet variance; a reduction in variance is observed when scaling range averaging is applied to the multitaper or structure function estimators.

The multitaper spectral, wavelet variance, and structure function estimators were applied to analyze turbulence data collected by an aircraft mounted platform. Extensive scaling ranges were observed where the SDFs and wavelet variances were well characterized by a linear approximation; the corresponding structure function regions were not well characterized by a simple linear relationship. In addition, the temperature related signals exhibited a leakage phenomenon related to a quasi-periodic component that contaminated the structure function across the entire range of scales; this same component was isolated at small scales in the wavelet variance and at high frequencies in the SDF. The structure function was clearly inferior to the other two approaches in providing a thorough analysis of the aerothermal series.

In this study we considered only data with Gaussian distributions; however, many physical processes, including turbulence, exhibit non-Gaussian behavior. A complete assessment of the effects of non-Gaussianity on these estimators is beyond the scope of this study; however, we conjecture that the wavelet approach should generally work well because, even if the data themselves are non-Gaussian, their wavelet coefficients (particularly those at large scales) will be approximately Gaussian due to a central limit effect from the averaging inherent in the MODWT (this argument breaks down in cases of marked nonGaussianity).

We recommend either the multitaper approach or the wavelet variance approach as the estimator of choice because of their ability to provide accurate estimates with appropriate confidence intervals. The wavelet variance has the best performance as long as the filter length is long enough to act as an adequate approximation to a band-pass filter. It is important to use the weighted least squares approach with the wavelet variance estimator to achieve this performance. The multitaper approach provides quality estimates with very 
little bias. The multitaper approach is relatively simple to implement and computationally efficient when a fast Fourier transform algorithm is used.

\section{Acknowledgments}

This work was supported by the U.S. Air Force Office of Scientific Research, Contract \#FA9451-04-M-0390, AM03.

\section{References}

[1] P. Abry, P. Gonçalvès and P. Flandrin, Wavelets, spectrum estimation, and $1 / f$ processes, Lecture Notes in Statistics 103; Wavelets and Statistics (A. Antoniadis and G. Oppenheim, eds.), (1995) 15-30.

[2] P. Abry, P. Flandrin, M. S. Taqqu and D. Veitch, Wavelets for the analysis, estimation, and synthesis of scaling data, Self-Similar Network Traffic and Performance Evaluation (K. Park and W. Willinger, eds.), (Wiley 2000) 39-88.

[3] R. A. Antonia and R. J. Smalley, Scaling Range Exponents from X-Wire Measurements in the Atmospheric Surface Layer, Boundary Layer Meteorology 100 (2001) 439-457.

[4] W. Constantine, D. B. Percival and P. G. Reinhall, Inertial range determination for aerothermal turbulence using fractionally differenced processes and wavelets, Physical Review E 64 (2001) 036301/1-036301-12.

[5] S. Corrsin, On the spectrum of isotropic temperature fluctuations in isotropic turbulence, Journal of Applied Physics 22 (1951).

[6] C. E. Coulman, J. Vernin and A. Fuchs, Optical seeing - mechanism of formation of thin laminae in the atmosphere, Applied Optics 34 (1995) 5461-5474.

[7] N. Cressie, Fitting variogram models by weighted least squares, Journal of the International Association for Mathematical Geology 17 (1951), 563586.

[8] M. G. Genton, Variogram fitting by generalized least squares using an explicit formula for the covariance structure, Mathematical Geology 30 (1998) 323-345.

[9] R. J. Hugo, S. R. Nowlin, I. L. Hahn, F. D. Eaton and K. A. McCrae, Acoustic noise-source identification in aircraft-based atmospheric temperature measurements, AIAA Journal 40 (2002) 1382-1387.

[10] M. J. Jensen, Using wavelets to obtain a consistent ordinary least-squares estimator of the long-memory parameter, Journal of Forecasting 18 (1999) $17-32$.

[11] A. N. Kolmogorov, Local structure of turbulence in an incompressible 
fluid at very high Reynolds numbers, Doklady Akad. Nauk SSSR 30 (1949) 299-303.

[12] E. J. McCoy and A. T. Walden and D. B. Percival, Multitaper spectral estimation of power law processes, IEEE Transactions on Signal Processing 46 (1998) 655-668.

[13] J. F. Muzy, E. Bacry and A. Arnèodo, Multifractal formalism for fractal signals: The structure-function approach versus the wavelet transform modulus maxima method, Physical Review E 47 (1993) 875-884.

[14] A. M. Obukhov, Structure of the temperature field in a turbulent flow, Izv. Akad. Nauk SSSR, Ser. Geograf. Geofiz. 131948.

[15] D. B. Percival, Simulating Gaussian random processes with specified spectra, Computing Science and Statistics 24 (1992) 534-538.

[16] D. B. Percival and A. T. Walden, Spectral Analysis for Physical Applications (Cambridge University Press, Cambridge, 1993).

[17] D. B. Percival and A. T. Walden, Wavelet Methods for Time Series Analysis (Cambridge University Press, Cambridge, 2000).

[18] K. S. Riedel and A. Sidorenko, Minimum biased multitaper spectral estimation, IEEE Transactions on Signal Processing 43 (1995) 188-195.

[19] D. J. Thomson, Spectrum estimation and harmonic analysis, Proceedings of the IEEE 70 (1982) 1055-1096.

[20] B. Vidakovic, Statistical Modeling by Wavelets (Wiley 1999)

[21] A. T. Walden, D. B. Percival and E. J. McCoy, Spectrum estimation by wavelet thresholding of multitaper estimators, IEEE Transactions on Signal Processing 46 (1998) 3153-3165.

[22] A. M. Yaglom, Correlation Theory of Stationary and Related Random Functions I; Basic Results (Springer-Verlag New York, Inc., New York, 1987) 